\title{
Circuit
}

Musiques contemporaines

CIRCUIT

\section{Entre liturgie et rituel}

\section{Feldman et Messiaen : dialogue imaginaire sur la spiritualité}

dans la musique

\section{Between liturgy and ritual}

Feldman and Messiaen : imaginary dialogue on sprituality in music

\section{Simon Bertrand}

Volume 21, numéro 1, 2011

Du spirituel dans l'art?

URI : https://id.erudit.org/iderudit/1001164ar

DOI : https://doi.org/10.7202/1001164ar

Aller au sommaire du numéro

\section{Éditeur(s)}

Les Presses de l’Université de Montréal

ISSN

1183-1693 (imprimé)

1488-9692 (numérique)

Découvrir la revue

Citer cet article

Bertrand, S. (2011). Entre liturgie et rituel : Feldman et Messiaen : dialogue imaginaire sur la spiritualité dans la musique. Circuit, 21(1), 75-79.

https://doi.org/10.7202/1001164ar
Résumé de l'article

La spiritualité en musique se limite-t-elle à la représentation d'un dogme et l'utilisation des diverses composantes de sa liturgie, ou s'agit-il aussi d'une certaine approche du son que l'on peut retrouver dans les stratégies compositionnelles du compositeur ? Telle est la question que veut poser cet article, où il est souhaité de générer, par l'interprétation de certains de leurs écrits, une sorte de dialogue imaginaire entre le " compositeur théologique " Olivier Messiaen et le « mystique agnostique » Morton Feldman. Il est aussi souhaité de réfléchir sur les rapports entre spiritualité et musique en général dans la musique $\mathrm{du} \mathrm{xx}^{\mathrm{e}}$ siècle, ainsi que sur les diverses implications qu'une quête spirituelle peut avoir sur la production et la pensée esthétique d'un compositeur, que celui-ci soit croyant ou non, et que cette quête se concrétise par des oeuvres dites " sacrées " ou " profanes ». 


\title{
Entre liturgie et rituel
}

\section{Feldman et Messiaen : dialogue imaginaire sur la spiritualité dans la musique}

\author{
SIMON BERTRAND
}

Qu'est-ce que la spiritualité en musique? Est-ce uniquement la représentation musicale d'un dogme, l'utilisation de ses symboles religieux, de ses écritures, des diverses composantes de sa liturgie, ou est-ce aussi une certaine approche du son, des stratégies compositionnelles du compositeur jusqu'à la perception de l'auditeur? En "opposant" deux approches aussi éloignées, tant sur le plan esthétique que dans la relation à la spiritualité, que celles du "compositeur théologique" Olivier Messiaen et du "mystique agnostique" Morton Feldman, il est souhaité ici de générer une sorte de dialogue imaginaire entre deux personnages marquants de la musique du xxe siècle.

\section{Morton Feldman}

The composer (...) doesn't have the problem of truth. What I mean is he doesn't work with the impossibility of ever reaching it, like the painter, or the poet. For the composer, the truth is always the process, the system.

Morton Feldman'

1. Feldman, in Zimmermann, 1976, p. 4-5.
Le "cas" de Morton Feldman (1926-1987) est troublant, puisque ce dernier a toujours soigneusement évité de parler de façon directe de l'aspect rituel et spirituel de sa musique, bien que plusieurs voient dans ses œuvres de longues méditations ou prières. Pour Feldman, l'aspect méditatif de ses œuvres n'est que la conséquence de son approche au phénomène sonore: My music sometimes seems mysterious. Part of the mystery comes from the fact that I wait, receptively, then I welcome, I accept... I feel that music should have no vested interests, that you shouldn't know how it's made, that you shouldn't know if there's a system, that you shouldn't know anything about it [...] except that it's some kind of life force that to some degree really changes your life ... if you're into it. ${ }^{2}$

Comme c'est souvent le cas, il y a dans ce propos de Feldman une bonne part de non-dit, de flottant, et même de contradictions qui portent à diverses interprétations. Quels sont précisément ces "vested interests" dont la musique devrait s'affranchir? Résident-ils dans le "message" d'une musique, ou dans ses intentions expressives? Et pourtant, dans le même entretien, 2. Feldman, 1967, p. 86 
Feldman accorde à la musique le statut de force de vie pouvant changer la nôtre. En tout cas, nous semblons ici déjà à des années-lumière de Messiaen et de son utilisation spécifique des symboles d'un dogme précis. Ici, c'est l'attitude du compositeur face au son qui relève d'un état religieux, et l'approche de Feldman, en ce sens, est peut-être beaucoup plus proche de la recherche d'une forme de fonction rituelle de la musique dans notre existence que de toute forme de représentation du religieux.

Et cette fonction rituelle n'est pas forcément compatible avec le contexte conventionnel du concert, d'où, peut-être, le malaise de Feldman face à celui-ci :

I'm looking for something else now, something that will no longer fit into the concert hall. If music would ever take that road, that direction, that would be the real composer's paradise... ${ }^{3}$

Il est intéressant de noter que ce malaise chez Feldman face au rituel du concert semble aujourd'hui plus que jamais d'actualité chez les nouvelles générations de compositeurs. N'y aurait-il pas en cela un retour de la recherche d'une forme de fonction rituelle de la musique dans notre société, en dehors des représentations d'éléments du culte ou de références spécifiques à un dogme, qui serait associée à une recherche de nouveaux lieux offrant une alternative aux salles de concert ou aux églises qui accueillent généralement les musiques "sacrées"?

Lieu ayant inspiré l'œuvre éponyme de Feldman, qui fut d'ailleurs conçue pour y être interprétée, la Rothko Chapel (1971) est peut-être un exemple de cette recherche. II s'agit à la fois d'un lieu de méditation et 3. Pickstock, 2008, p. 176. de prière sans aucune dénomination religieuse précise, ouvert à toutes les croyances, d'où est absente toute forme de symbole religieux, et d'un espace d'exposition, où l'on retrouve les fameuses toiles du peintre Mark Rothko. L'œuvre de Feldman, pour soprano, double chœur et trois instruments, se déploie de manière extrêmement lente, avec de longs accords tenus au chœur, enluminés de colorations instrumentales, laissant à l'auditeur un grand espace d'introspection et de contemplation.

Une question se pose alors: la représentation de symboles religieux ou l'utilisation d'éléments spécifiques du culte en musique équivaut-elle à une quête de transcendance? Inversement, la spiritualité en musique est-elle uniquement l'affaire de la musique "sacrée" ?

En effet, parallèlement à la tradition de la musique sacrée occidentale, généralement associée directement à des éléments liturgiques (célébration du culte, ou mise en musique de ses écrits), il existe dans de nombreuses sociétés extra-occidentales des pratiques musicales ayant une fonction rituelle purement associée à des éléments du quotidien, sans nécessairement être, à proprement parler, associée aux constituants spécifiques d'un dogme ou des pratiques d'un culte, même si cette distinction est moins évidente dans des sociétés où la frontière entre "vie quotidienne" et "vie spirituelle" n'est pas aussi délimitée qu'elle tend à l'être dans les sociétés occidentales...

Pourtant, la présence de la musique dans notre quotidien, et l'aspect rituel qu'elle peut assumer, peuvent nous conduire à la contemplation, à la méditation et à l'introspection tout autant qu'une musique liturgique. 
De même, il serait restrictif, voire présomptueux, d'affirmer que seuls les croyants qui adhèrent à un dogme spécifique peuvent avoir une telle relation avec la musique. Au contraire, la spiritualité, les croyances et, surtout, la foi sont des choses à la fois extrêmement personnelles et communes; elles se vivent de diverses manières et peuvent se rattacher ou pas à un dogme. Dans cet esprit, il apparaîtrait donc simpliste, voire sectaire, de ranger la musique dans deux catégories, l'une "sacrée", l'autre "profane". Cela reviendrait à lui nier toute forme de spiritualité n'étant pas associée directement à un dogme. Après tout, un mélomane ne peut-il pas être autant transporté spirituellement par une simple sonatine pour piano de Mozart que par son Requiem?

\section{Olivier Messiaen}

Asked whether he was a "mystical composer," Olivier Messiaen denied this and replied that he was a theological composer. This comment is elucidated by Messiaen's other statement that, for him, music provides a more rather than less exacting means of saying things than the words of language.

Catherine Pickstock ${ }^{4}$

À première vue, il ne semble y avoir de contraste plus violent que lorsqu'on compare la démarche de Feldman, imbibée de ce que nous pourrions qualifier d'une forme de "mysticisme agnostique", à celle d'Olivier Messiaen, compositeur croyant et «théologique", avec ses représentations directes des diverses constituantes du catholicisme, de ses symboles et des éléments liturgiques. Lorsque Messiaen va jusqu'à identifier clairement dans une partition un Thème de Dieu, 4. Pickstock, 2008, p. 176. un Thème des anges, un Battement du cœur de Jésus, il affiche clairement ses intentions et sa foi. Nous sommes alors, en effet, très loin du flou soigneusement entretenu par Feldman quant aux intentions spécifiques de sa musique.

Par contre, ce que l'article de Pickstock, cité plus haut, souligne très justement, c'est que pour Messiaen, nonobstant la présence d'une symbolique religieuse on ne peut plus clairement représentée, c'est la recherche de l'expression par la musique de ce que les mots ne peuvent, selon lui, aussi bien évoquer. L'expression de la spiritualité et de ses divers états, de la quête de l'illumination, trouve donc pour Messiaen son expression d'abord et avant tout dans le son.

Sommes-nous, alors, toujours aussi loin de Morton Feldman? C'est ici que se pose la question délicate du témoignage de la foi. Si un compositeur adhère aux préceptes spécifiques d'une religion, quelle qu'elle soit par ailleurs, rien ne l'oblige a priori à le dévoiler dans sa musique, sinon un désir de témoigner, voire de transmettre sa foi, ou alors de rendre grâce par son œuvre. Mais alors, le compositeur croyant qui prend cette décision ne risque-t-il pas de repousser l'oreille du non-croyant? Chez Messiaen, l'admiration de la nature semblait constituer le lien lui permettant de rattacher son témoignage de foi à des éléments auxquels le noncroyant pouvait aussi se rattacher:

Pour ma part, j'écris des œuvres musicales religieuses qui sont des actes de Foi mais qui contiennent aussi mon admiration de la nature par l'utilisation des chants d'oiseaux et de nombreuses allusions aux différentes étoiles de notre galaxie 5 .

5. www.musimem.com/messiaen.htm 
Mais le désir de faire acte de foi ne va pas de soi pour tous les compositeurs croyants, et lorsque certains compositeurs font ce choix, il n'est pas systématiquement appliqué à toutes leurs œuvres, comme le démontre d'ailleurs le catalogue d'autres compositeurs croyants comme Claude Ballif ou Gilles Tremblay, pour n'en nommer que deux.

Mais le compositeur n'a-t-il pas besoin de temps à autre de se nourrir de recherche "pure" avant de pouvoir aspirer à la transcendance? Messiaen a mentionné:

J'ai écrit des musiques pures (pour la seule recherche technique) ou à caractère profane. Je les regrette presque. Les musiques créées pour chanter les mystères de la Foi me paraissent plus utiles pour mes contemporains ${ }^{6}$.

Messiaen, pour sa part, a su habilement se libérer du carcan ancien des formes de la musique sacrée, en choisissant soigneusement, à l'intérieur du catholicisme, une symbolique qui le stimulait, sans recréer des formes du passé, et en assignant ces éléments à des leitmotive d'une manière sensiblement wagnérienne (bien différente, par exemple, de l'approche de son contemporain Maurice Duruflé).

L'œuvre entière de Messiaen est une sorte de grand "opéra théologique", où il met librement en scène cette riche symbolique, qu'il offre autant au croyant qu'au non-croyant. Un exemple particulièrement représentatif de cette attitude se trouve dans la TurangalîlaSymphonie, où la foi catholique de Messiaen et son amour de la vie terrestre semblent en parfaite harmonie. Le résultat est une œuvre à la fois profondément humaniste et spirituelle.

6. Cité in Meltzheim et Ide, 1991, p. 24.

\section{Et nous...}

In modern times, one could argue, music has tended to become a substitute for religion. If, on hearing music, one is bound in some sense to intimate cosmic harmonies, then it seems nonetheless possible to halt at a certain mystical agnosticism which will not countenance doctrinal formulae or metaphysical explanations.

Catherine Pickstock ${ }^{7}$

Il apparaît évident que la musique "sacrée", avec ses représentations des symboliques religieuses et des composantes de la liturgie, a été, outre quelques exceptions, un peu mise de côté pendant la deuxième moitié du $x x^{e}$ siècle. Est-ce à dire qu'il n'y a plus de recherche et de quête spirituelle dans le travail des compositeurs? Ou est-ce tout simplement que cette recherche spirituelle, pour des raisons historiques telles que la recrudescence des conflits entre religions et l'hyper-individualisation des démarches spirituelles, a pris d'autres formes chez les compositeurs?

Les compositeurs de la fin du $x^{e}$ siècle témoignent peut-être à leur façon, par une forme de désintérêt envers un certain genre de musique sacrée telle qu'elle a existé pendant quelques siècles, d'un monde où la recherche spirituelle est à la mode, mais où les dogmes, eux, sont démodés. Mais cela ne veut pas dire pour autant que leur musique n'est plus porteuse de quête spirituelle.

Par ailleurs, des titres évocateurs présents dans la production des compositeurs depuis une cinquantaine d'années (Répons, Offertorium, Rituel...) semblent suggérer que certaines formes de représentation de la spiritualité sont toujours bel et bien présentes dans les œuvres nouvelles, mais que les compositeurs, témoins 
de leur époque, semblent réticents, qu’ils soient croyants ou non, à perpétuer une certaine forme de musique sacrée associée directement au culte et à sa liturgie. Bien sûr, on ne peut évidemment pas se fier uniquement aux titres évoquant la présence de formes musicales liturgiques pour délimiter dans la production des compositeurs les pièces qui sont ou ne sont pas porteuses de spiritualité. Par contre, il est certain que le public peut être, d'une certaine manière, mis en contexte par un titre évocateur, ou une référence à une forme liturgique, ou l'utilisation de textes sacrés, et qu'il aborde sans doute ces œuvres avec des attentes et une écoute différentes que pour une œuvre simplement intitulée Pièce pour piano..

Si on considère, que l'on soit croyant ou non, que la musique peut élever l'âme et que celle-ci peut être associée à des moments de contemplation, de prière et de méditation, alors le compositeur souhaitant que sa musique aille dans cette direction est d'abord et avant tout confronté à une recherche concrète sur le plan des stratégies compositionnelles. Si, comme le disait Victor Hugo, "la musique commence là où les mots s'arrêtent", alors qu'importe le titre ou la présence en filigrane de mots évocateurs, c'est bel et bien le son qui doit créer un espace fertile pour une chose aussi intime que la quête spirituelle.

Finalement, il ne faudrait pas négliger le malaise possible de toute une génération de compositeurs peutêtre un peu désabusés face aux conséquences fâcheuses de l'extrémisme religieux. Si la religion a peut-être, aujourd'hui, plutôt mauvaise réputation chez certains, la quête de l'humain vers la transcendance à travers le son ne s'est pas arrêtée pour autant. Peut-être s'est-elle tout simplement transformée, en attendant des temps meilleurs pour le dogme. Mais que le compositeur décide ou pas de composer une œuvre en se basant sur des éléments liturgiques ou de témoigner de sa foi par sa musique, il ne sera jamais dispensé de sa recherche compositionnelle et de ce rapport intime au son par lequel il tente de trouver l'expression juste de sa quête intérieure. Et cette recherche est, sans jeu de mots, un travail de moine... dans une tour de Babel.

\section{B I B LIOGRAPHIE}

Feldman, Morton (1967), «Conversations without Stravinsky ", London Magazine, vol. 6, n 12, p. 86-94; rééd. in Give my regards to Eighth Street: collected writings of Morton Feldman, B.H. Friedman (éd.), Cambridge, MA, Exact Change, 2000, p. 50-62.

Meltzheim, Irène et IDE, père Pascal (1991), «Le musicien de la joie », entretien avec Olivier Messiaen, in Du côté de la Trinité, journal de la paroisse de la Trinité, $\mathrm{n}^{\circ} 2$ de mars 1991, repris in Musica et Memoria, $\mathrm{n}^{\circ} 42$ de juin 1991, p. 22-27.

Pickstock, Catherine (2008), «Messiaen and Deleuze: the musico-theological critique of modernism and postmodernism ", Theory, Culture and Society, 25 (7-8), p. $176-181$.

Zimmermann, Walter (1976), Desert Plants: Conversations with 23 American Musicians, Vancouver, A. R. C. Publications, p. 4-5. 